\title{
CARTOGRAFÍA DE AFECTACIÓN POR AMENAZAS NATURALES EN EL CANTÓN DE POÁS, COSTA RICA
}

\author{
AFFECTATION CARTOGRAPHY FOR NATURAL \\ HAZARDS IN POÁS CANTON, COSTA RICA
}

\author{
Gustavo Barrantes Castillo ${ }^{1}$ \\ Adolfo Quesada Román ${ }^{2}$ \\ Universidad Nacional, Costa Rica
}

\begin{abstract}
RESUMEN
A pesar de la existencia de reportes históricos, recopilaciones, bases de datos e informes, no se dispone en el país de un estudio que sintetice y distribuya espacialmente la información sobre afectación histórica por amenazas naturales. Por medio de una compilación y georreferenciación de toda la información disponible sobre impactos por amenazas naturales en el cantón de Poás, fue posible construir indicadores espaciales de afectación para los eventos registrados (caída de ceniza, gases volcánicos, terremotos, inundaciones y deslizamientos). Estos fueron estandarizados en cinco clases (muy baja, baja, moderada, alta y muy alta), con lo que se construyó un indicador de máxima afectación por eventos naturales históricos para dicha unidad política-administrativa a partir de la sobreposición de los registros de desastres. El resultado de este estudio muestra una tendencia al incremento de las afectaciones por amenazas naturales de sur a norte, lo que es consecuente con la distribución de las lluvias, las pendientes, así como la proximidad con fuentes sísmicas y volcánicas. Un patrón distinto mostraron las inundaciones rápidas y los deslizamientos que se relacionan con la presencia de valles fluviales encañonados con fuertes pendientes en microcuencas alargadas y pequeñas sobre depósitos volcánicos recientes.
\end{abstract}

Palabras clave: Amenazas naturales, afectación por desastres, cantón de Poás, desastres, volcán Poás

1 Escuela de Ciencias Geográficas, Universidad Nacional, Costa Rica. gbarrantes@una.cr

2 Escuela de Ciencias Geográficas, Universidad Nacional, Costa Rica. adolfo.quesada.roman@una.cr

Fecha de recepción: 02 de marzo de 2016

Fecha de aceptación: 14 de junio de 2016 
Gustavo Barrantes Castillo, Adolfo Quesada Román. Affectation cartography for

natural hazards in Poás canton, Costa Rica

\begin{abstract}
Despite the existence of historical reports, compilations, databases and reports in Costa Rica that summarize zones affected by natural hazards since colonial times, there is not well-located historical information available in the country. Through a compilation and georeferencing of all the available information regarding impacts of natural hazards that affected the Poas canton, it was possible to create spatial indicators of the recorded events (ash falling, volcanic gases, earthquakes, floods, and landslides). The different natural hazards were standardized in five classes (very low, low, moderate, high and very high), which allowed creating a maximum impact indicator overlapping the disaster records of Poas canton. The results of this study show a tendency to generate increased impact by natural hazards from south to north, which is consistent with the distribution of rainfall, slopes, and proximity to seismic and volcanic sources. These outcomes also showed a different pattern in flash floods and landslides related to the presence of river valleys with steep slopes in elongated and small basins on recent volcanic deposits.
\end{abstract}

Keywords: Natural hazards, disaster affectations, Poás canton, disasters, Poás volcano.

\title{
Introducción
}

La calidad de la información sobre el riesgo está directamente relacionada con el tipo de información que se quiere para hacer gestión, en cuanto a las amenazas los datos sobre desastres históricos son de amplia utilidad en aspectos sociales y económicos (Ordaz, 2007). No obstante, en Costa Rica no se cuenta con un registro de desastres que reúna los requisitos para comprender el comportamiento espacial y temporal de los mismos y así como para su uso en la modelación de amenazas naturales.

Un registro estandarizado, sistemático y georreferenciado se justifica en la posibilidad de utilizarlo para caracterizar el comportamiento de los eventos potencialmente destructivos, con miras a la comprensión de sus patrones espaciales, las posibles interacciones entre estos y el modelamiento de su comportamiento. Si bien se han hecho esfuerzos para sistematizar registros históricos y actuales, apenas se ha implementado la ubicación de los mismos en unidades político-administrativas o de cuencas hidrográficas, en el caso de informes actuales, se brinda una ubicación imprecisa o especulativa y en pocas ocasiones por coordenadas geográficas.

Con la finalidad de representar espacialmente las áreas de afectación por eventos naturales a nivel territorial, vinculados con desastres, en la presente investigación se consultaron las fuentes disponibles en Costa Rica, con el objetivo de construir un indicador de afectación por múltiples amenazas. Como caso de estudio se ha escogido el cantón de Poás, debido a que ha sido afectado por varias amenazas naturales como: la sismicidad, el vulcanismo, las inundaciones rápidas y los deslizamientos, que incluso han interactuado entre sí para provocar eventos en cadena, como ocurrió con el terremoto de Cinchona en 2009 (Barrantes, Jiménez, \& Ocón, 2013). 


\section{Área de Estudio}

El cantón de Poás (Mapa 1) fue creado en el Decreto $\mathrm{N}^{\circ} 14$ del 15 octubre de 1901, es el octavo cantón de la provincia de Alajuela y está dividido en cinco distritos: San Pedro, San Juan, San Rafael, Carrillos y Sabana Redonda. Además, su extensión es de $73,84 \mathrm{~km}^{2}$, y su población era de 29199 personas según el censo de 2011 (INEC, 2012).

Mapa 1. Localización geográfica del cantón de Poás.

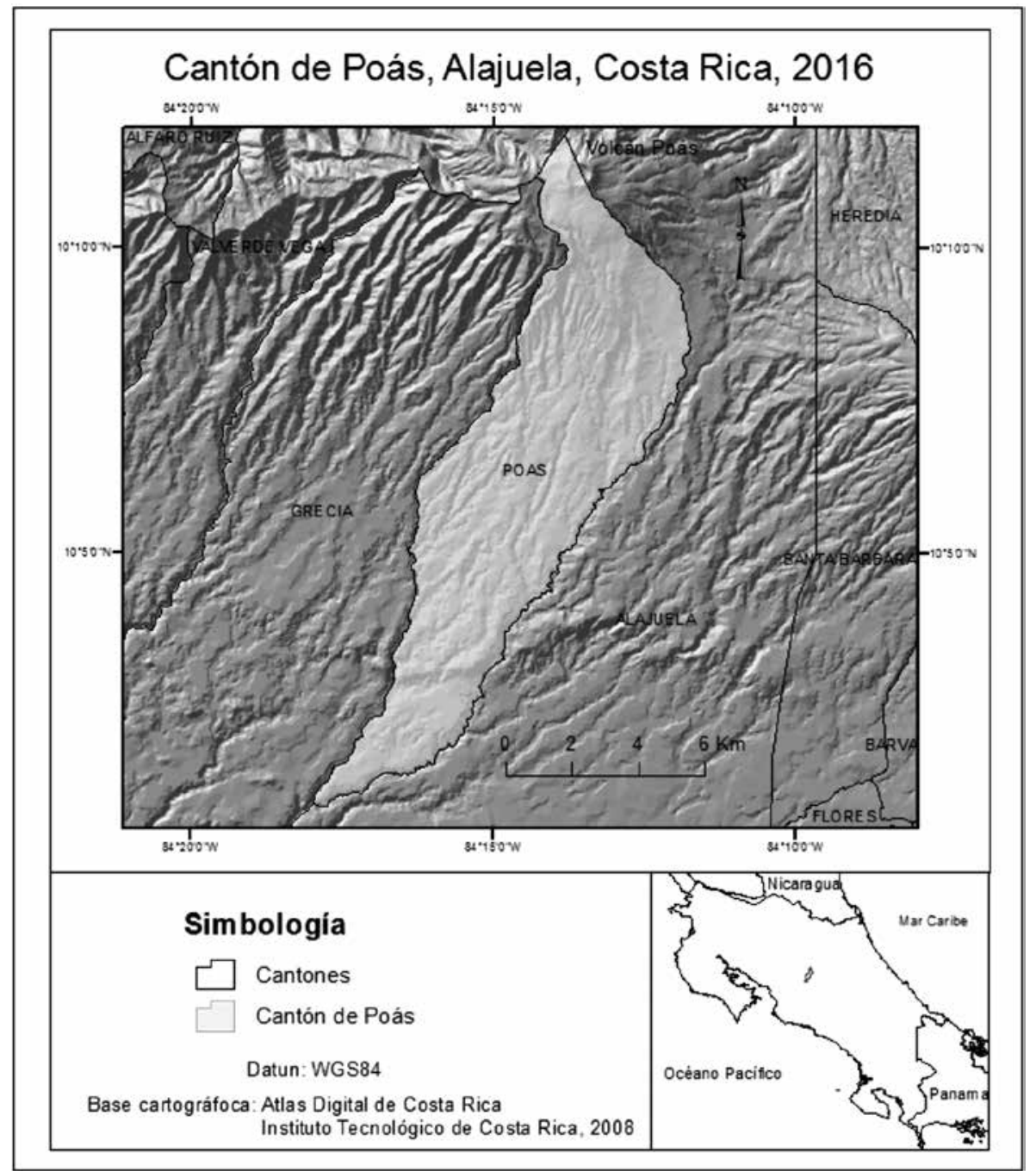

Fuente: elaboración propia 
De acuerdo con el Atlas de amenazas naturales de Costa Rica de la Comisión Nacional de Atención de Emergencias y Prevención de Desastres (CNE, 2008), el cantón de Poás presenta una serie de amenazas naturales entre las que se destacan las siguientes:

\section{Amenaza Sísmicas}

En las proximidades del cantón, existen fuentes sísmicas importantes como: la falla El Ángel - Varablanca y la Fallas Viejo - Aguas Zarcas (CNE, 2008). En los alrededores del Volcán Poás, han ocurrido sismos históricos destructivos tales como: el sismo del 15 de febrero de 1772, el del 30 de diciembre de 1888, el del 28 de agosto de 1922, el del 6 de junio de 1912 , el del $1^{\circ}$ de septiembre de 1955 y el reciente terremoto del 8 de enero del 2009, todos los anteriores, relacionados con las fallas Ángel, Sabanilla y Viejo - Aguas Zarcas (Montero et al, 2010).

\section{Amenaza volcánica}

La zona de estudio se ubica en la ladera sur del Volcán Poás, contempla parte del cráter principal y la laguna Botos. En caso de una erupción volcánica, los efectos sobre el cantón serían (CNE, 2008):

- Caída de ceniza, lo que causaría contaminación de las fuentes de agua, pérdidas de cultivos y ganado.

- Emanación de gases, los cuales afectarían sobre todo los sectores al norte del cantón, causando trastornos respiratorios a la población, además de daños a cultivos y la actividad ganadera.

Adicionalmente, el macizo del Poás es atravesado en sentido nortesur por una zona de fractura vulcano-tectónica con presencia de conos piroclásticos monogenéticos y coladas de lava (Gazel \& Ruiz, 2005). A pesar de que estos conos son antiguos se debe considerar el potencial de aparición de nuevos conos sobre esta zona de debilidad, así como de coladas de lava.

\section{Amenaza por remoción en masa}

De acuerdo con la Comisión Nacional de Prevención de Riesgos y Atención de Emergencias (CNE) el cantón de Poás es susceptible a los 
movimientos en masa, particularmente, al norte del cantón (CNE, 2008), dada la fuerte pendiente, la alta precipitación y la presencia de gruesos suelos volcánicos en este sector.

\section{Los inventarios de desastres para Costa Rica}

Los inventarios sobre el impacto de eventos naturales disponibles en Costa Rica son estudios descriptivos a manera de recopilaciones históricas como el documento elaborado por Cleto González Víquez en 1910, donde reúne datos sobre eventos ocurridos entre 1608 y 1910. Por su parte, Montero \& Salazar (1991) y más recientemente, el trabajo de Vallejos et al. (2012) realizan recopilaciones históricas en secuencia cronológica por tipo de evento desplegado en un cuadro resumen con los efectos más relevantes. Los trabajos que se aproximan a la localización y realizan una caracterización de las condiciones fisiográficas de sitio, para el caso de Costa Rica, son los trabajos de Arroyo \& Patterson (1988) y Arroyo \& Lavell (1991), en donde se aproxima la ubicación por cuencas hidrográficas de eventos tales como los deslizamientos e inundaciones, este procedimiento es luego retomado y ampliado en Vahrson et al. (1990) para el tema de las inundaciones, en todos estos estudios la fuente de información son los registros periodísticos.

Por otro lado, existen bases de datos alfanuméricas que mantienen un registro de eventos ocurridos en Costa Rica, con base en reportes de prensa, donde destaca a nivel mundial "The International Disaster Database" del Centro para la investigación en epidemiología y desastres (CRED por sus siglas en inglés) (EM-DATA, 2015), el cual mantiene un registro usando como criterio de selección si el evento tuvo más de 10 muertos, más de 100 personas afectadas, una declaratoria de emergencia nacional o petitoria de asistencia internacional. También existe el Sistema de Inventario de Efectos de Desastres (DesInventar), base de datos que mantiene un registro de afectaciones ocurridos en América Latina y otras zonas del mundo, este sistema permite consultar por tipo de amenaza a escala de municipio e incluso está disponible a nivel de distrito para Costa Rica desde 1970 (LA RED, 2015).

En cuanto a bases de datos espaciales, destaca la de la CNE, que resulta ser un repositorio de estudios de distintas calidades y escalas espacio-temporales, que en ocasiones se superponen, así como de mapeos 
Gustavo Barrantes Castillo, Adolfo Quesada Román. Affectation cartography for natural hazards in Poás canton, Costa Rica

parciales e imprecisos de afectaciones por eventos naturales o desastres particulares (Una vista de parte de la base de datos de la CNE puede apreciarse en el Mapa 2). Su mayor limitación está en la ausencia de información alfanumérica que acompañe las capas gráficas, así como de metadatos; en términos prácticos, esto impide asociar información relevante a una amenaza; por ejemplo, conocer el nombre de una falla, si se encuentra activa, si se le asocia con un terremoto, o la altura alcanzada por el nivel de las aguas durante una inundación particular.

Mapa 2. Síntesis de amenazas naturales de Costa Rica.

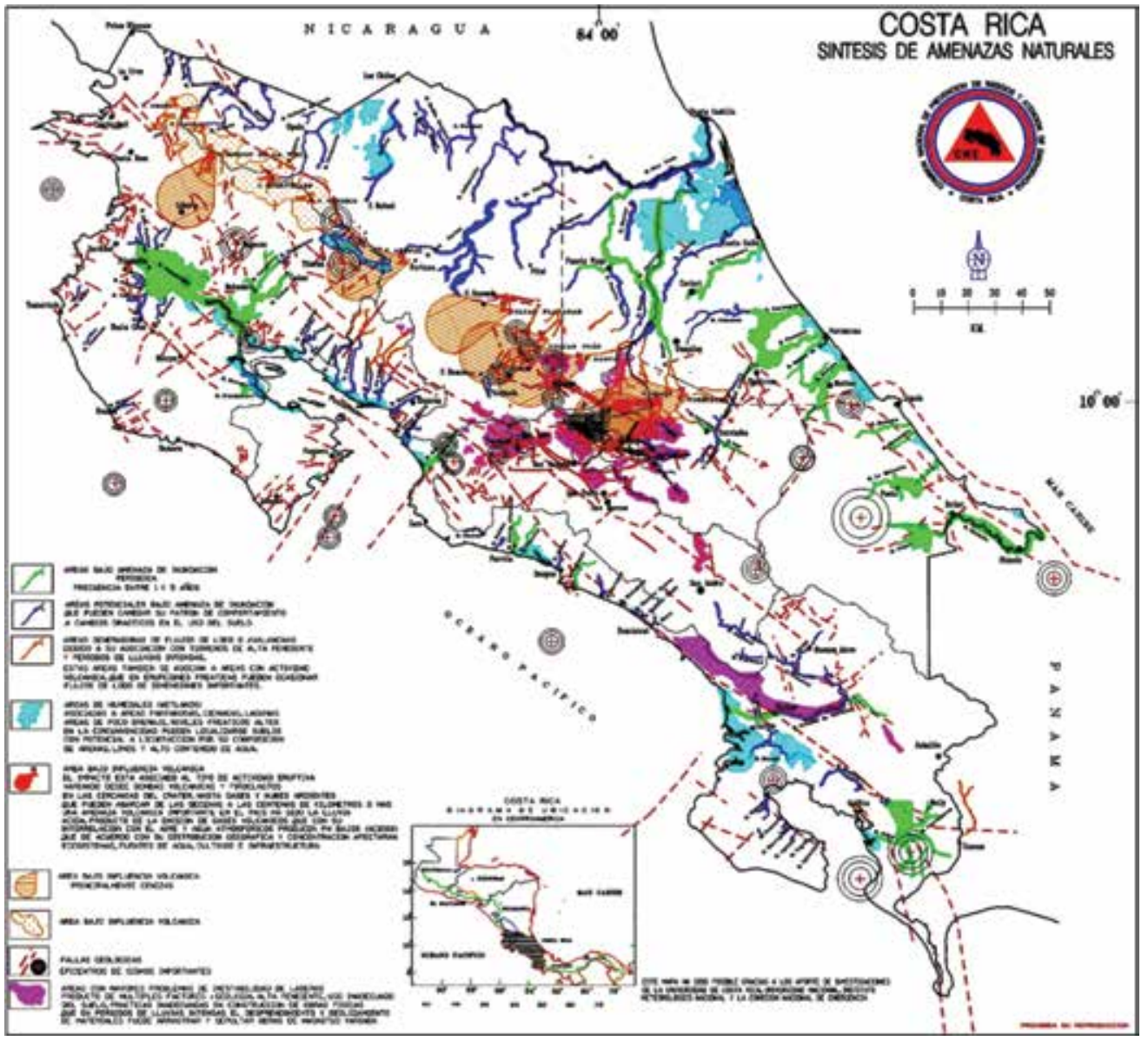

Fuente: CNE, 2008. 


\section{Metodología}

Luego de revisar la información existente para el caso de Poás (mencionados en el apartado anterior), se determina que es posible construir mapas individuales de afectación para las siguientes amenazas naturales: caída de ceniza, gases volcánicos, terremotos, inundaciones y deslizamientos.

El primer paso consistió en la revisión de cada una de las fuentes de información con el fin de construir un cuadro resumen con los siguientes datos: tipo de evento, fecha, ubicación, daños o grado de afectación, formato de la información, fuente y observaciones. Este paso implicó la consolidación de los datos para evitar repeticiones y complementar la información suministrada por distintas fuentes.

En segundo lugar, se georreferenciaron los reportes de afectación de las distintas fuentes, estos se puede visualizar en el cuadro mencionado anteriormente. Para este fin se utilizó un Sistema de Información Geográfica (SIG), por medio del cual se digitalizaron las áreas de afectación reportadas en mapas o figuras, de acuerdo con las fuentes. Por su parte, en los casos donde la ubicación no estaba acompañada de mapas o referencias a áreas, se optó por el uso de puntos, a los cuales se les asocia la caracterización del evento vinculando, por medio de un cuadro de atributos realizado con anterioridad (cuadro resumen).

Cuando la información existente lo permitió, se clasificó el grado de daño o afectación, para cada evento individual, en cinco categorías; a saber: muy baja, baja, moderada, alta y muy alta. Posteriormente, se sobreponen las áreas de afectación de todos los eventos del mismo tipo de amenazas y se definen zonas de afectación con las mismas cinco categorías, pero en esta ocasión los polígonos resultantes asumen el valor de la mayor afectación de entre los eventos que se sobreponen (Figura 1). En otras palabras, cuando dos o más eventos se sobreponen se utiliza la categoría más alta como el valor de máxima afectación histórica por un determinado tipo de evento, dentro del cantón. 
Gustavo Barrantes Castillo, Adolfo Quesada Román. Affectation cartography for natural hazards in Poás canton, Costa Rica
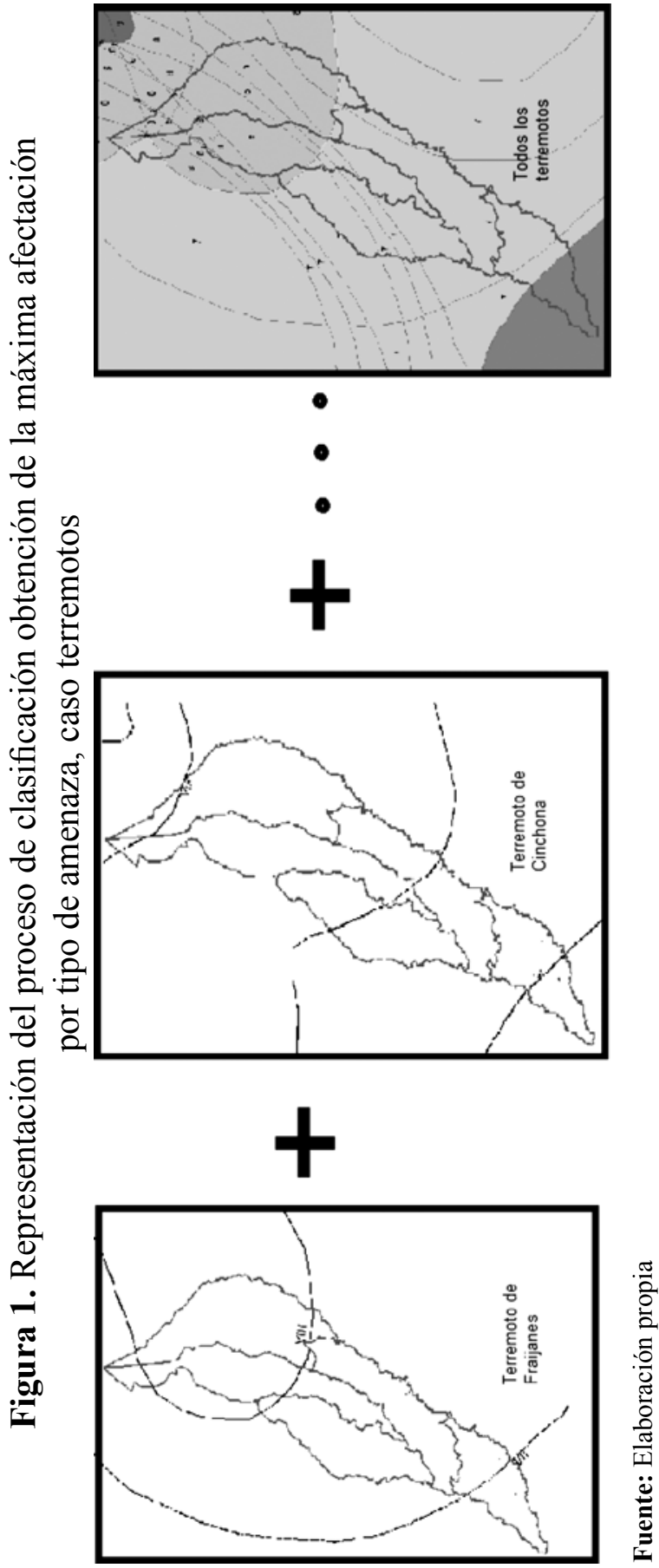

136 Revista Geográfica de América Central No 57 ISSN 1011-484X, julio-diciembre 2016, pp. 129-157 
Cuando no se dispuso de información suficiente para establecer el grado de severidad de la afectación, se optó por utilizar la cantidad de eventos sobrepuestos (para cada tipo de amenaza) como criterio de clasificación, asumiendo que la afectación frecuente involucra un mayor daño acumulado, este fue el caso del tratamiento utilizado con la amenaza volcánica.

Este procedimiento se repitió para cada tipo de amenaza específica (caída de ceniza, gases volcánicos, terremotos, inundaciones y deslizamientos). La comparación de todas las tipologías de eventos se logró por medio de la estandarización por clasificaciones en cinco categorías de afectación (muy alta, alta, moderada, baja y muy baja), de este modo fue posible combinar las afectaciones independientemente del tipo de amenaza que la originó. La máxima afectación histórica por amenazas naturales presente en cada sección del territorio se obtiene resaltando la mayor afectación registrada para todos los eventos.

A continuación, se describe el procedimiento específico y las fuentes de información utilizadas para cada uno de los siguientes tipos de amenazas: la caída de ceniza, los gases volcánicos, los terremotos, las inundaciones y los deslizamientos.

\section{Afectación por actividad volcánica}

En la elaboración del mapa de afectación por caída de ceniza se utilizaron los registros sobre la actividad volcánica procedente de la recopilación que realizara Cleto González Víquez en 1910 (González-Víquez, 1910), el trabajo de tesis de maestría de Raúl Mora (Mora, 2010) y los registros del Observatorio Vulcanológico y Sismológico de Costa Rica (OVSICORI) (Barquero \& Duarte, 1990).

Esta información fue localizada espacialmente y clasificada en cinco categorías, con base en la frecuencia espacial de afectación, bajo el principio de que las zonas que son constantemente cubiertas por eventos individuales representan áreas de mayor afectación y viceversa. El mismo procedimiento se utilizó para el caso de los gases volcánicos, donde se usaron los registros de OVSICORI (Barquero \& Duarte, 1990) y de la CNE (CNE, 2015) a partir de periodos eruptivos ocurridos entre 1984 y 1994. 


\section{Terremotos}

Para la elaboración del mapa de afectación máxima por terremotos, se utilizaron los mapas de isosistas máximas extraídos de los trabajos de Montero et al. (2010), Morales et al. (1992), Rojas et al. (2009) y RSN (2009). Fue necesario también integrar los mapas de isosistas de los terremotos de Fraijanes de 1888 y el de Cinchona de 2009, cada mapa de isosistas fue clasificado acorde con lo establecido en el cuadro 1.

Cuadro 1. Valoración de la afectación por sacudida sísmica en función de valores de Intensidad de Mercalli Modificada (IMM).

\begin{tabular}{|c|c|c|c|c|}
\hline $\mathbf{1}$ & $\mathbf{2}$ & $\mathbf{3}$ & $\mathbf{4}$ & $\mathbf{5}$ \\
\hline$\leq \mathrm{V}$ & $\mathrm{VI}$ & $\mathrm{VII}$ & VIII & $\geq \mathrm{IX}$ \\
\hline
\end{tabular}

Fuente: elaboración propia

Los mapas clasificados para cada evento fueron sobrepuestos, dejando la máxima afectación registrada como la categoría de afectación máxima por sismicidad.

\section{Inundaciones rápidas}

La pobre referencia espacial disponible para el caso de inundaciones rápidas no hizo posible su ubicación precisa ni su clasificación en grados de severidad, tan solo fue posible su vinculación con los cursos fluviales dentro de los límites del cantón. Los registros consultados en este caso fueron la compilación de Vahrson et al. (1990), la base de datos de DesInventar y los informes técnicos de la CNE.

De acuerdo con la base de datos de desastres DesInventar (LA RED, 2015), entre los años de 1999 a 2013 se reportaron 30 fichas relacionadas con eventos de inundación o lluvias extremas. Una vez leídos e interpretados estos datos, se procedió a realizar una aproximación espacial al relacionar su ubicación con los valles fluviales más próximos.

\section{Deslizamientos}

En el caso de los deslizamientos, la información disponible tampoco permite discriminar en grados de afectación y en el $80 \%$ de los casos solo fue posible acceder a la ubicación de deslizamientos. Dado que se trata de 
supreficies de ruptura o laderas con presencia de deslizamientos activos, se decidió clasificarlos como "muy alta" afectación, a partir del supuesto de que se trata de una masa que ya experimentó un movimiento, lo que reduce su estabilidad ante un nuevo evento disparador. "Cuando ha existido un movimiento de ladera en un lugar determinado, siempre existirá la posibilidad de que éste se reactive" (CENAPRED, 200, p14).

Las fuentes utilizadas en este caso fueron: la Base de Datos Espacial de la CNE (CNE, 2015), la hoja geomorfológica Abra 1: 50 000, el mapa de deslizamientos del Gran Área Metropolitana (Denyer \& Kussmaul, 1994), los eventos reportados por Barrantes (2013) para el Terremoto de Cinchona y los eventos identificados a partir de la revisión del MDT (Modelo de Elevación Digital) y del ortomosaico de fotografías aéreas de la zona de estudio. Cabe resaltar la reubicación realizada de los deslizamientos aportados por la CNE debido al detalle del MDT disponible.

Como en el caso de los deslizamientos, los flujos de detritos aportados por la CNE solo permiten su ubicación, no brindan información complementaria que oriente su clasificación por severidad. Dado que estos eventos suelen arrasar o dañar severamente lo que se encuentran a su paso, se optó por asignarles la categoría muy alta a todos.

\section{Resultados}

A continuación se presentan los resultados obtenidos con base en la información recopilada por tiempo de evento. Se inicia con las afectaciones volcánicas, luego las sísmicas, por inundaciones rápidas así como lahares y finalmente, por deslizamientos. Cuando la información lo permite, se estandariza en cinco categorías de severidad en relación con la intensidad o frecuencia con que se presenta el evento, acorde con lo establecido en la sección anterior. No obstante, en el caso de las inundaciones se utilizó un ícono que representó la ubicación aproximada de cada evento reportado con puntos de desborde de ríos, y para el caso de los deslizamientos solo fue posible ubicar los activos sin poder determinar su impacto o frecuencia.

\section{Afectación por Actividad volcánica}

De acuerdo con Prosser y Carr (1987) el Poás ha producido explosiones vulcanianas y estrombolianas leves y actividad regular desde el siglo pasado, caracterizada por emisiones de gases y ocasionales erupciones 
freáticas. Según Mora (2010) la mayor erupción histórica ocurrió en el periodo eruptivo 1953-55 con una columna eruptiva estimada en $5000 \mathrm{~m}$. Entre los peligros reportados se encuentra: la caída de cenizas, proyección balística, gases volcánicos, lluvia ácida, y lahares (Mora, 2010), así como emanaciones de lava como las confinadas al cráter principal reportados por Prosser \& Carr (1987). Sin embargo, dado el poco detalle existente en la información histórica, en particular, los eventos anteriores a la instrumentalización de los datos, solo se evaluará la afectación por caída de ceniza y gases volcánicos.

Cantones como: Poás, Valverde Vega, y Alajuela, se han visto afectos de manera directa por caída de tefra y en la zona proximal del cráter activo por lava y oleadas piroclásticas (Mora, 2010). En el caso de Grecia, Alfaro Ruíz, Naranjo se han visto afectados por lluvia ácida como en los eventos de 1968, 1989 y 1994 (RSN: UCR-ICE, 2014) y por cenizas como lo ocurrido en 1834, 1910, 1914-15 y 1955 (Vargas, 1979; Casertano et al., 1983).

De acuerdo con los resultados para el caso de caída de ceniza, en el cantón de Poás las zonas con mayor afectación se localizan en las cercanías del cráter principal, sobre los distritos de San Juan y Sabana Redonda (Mapa 3). Por su parte los distritos de Carrillos, San Pedro y San Rafael han presentado una afectación de moderada a baja para los eventos ocurridos entre 1910 y la actualidad. 
Mapa 3. Indicador de afectación por caída de ceniza



Fuente: elaboración propia 
En el caso de la afectación por gases volcánicos, se marca un clara tendencia con rumbo $\mathrm{W}$ desde el cráter principal, donde se localiza una área alargada sin vegetación que es debida al efecto combinado entre la topografía del terreno y la dirección principal de los vientos alisios, dicha área se produce a consecuencia de la alta concentración de los gases volcánicos en las cercanías del foco volcánico y es denominada por algunos autores como "callejón de acidificación” (Duarte \& Fernández, 2011). No obstante, cuando los eventos son mayores a lo habitual (columnas de gases de más de $500 \mathrm{~m}$ ) escapan al confinamiento topográfico y se dirigen al SW tendencia que se registra en Mapa 4.

Como sería de esperar, las zonas de afectaciones altas y moderadas se corresponden con la parte de mayor altitud de los distritos de Sabana Redonda así como San Juan y cambia rápidamente a afectaciones bajas hacia el centro de dichos distritos a consecuencia de la dirección predominante de los vientos.

Diversos gases como hidrógeno $\left(\mathrm{H}_{2}\right)$, dióxido de carbono $\left(\mathrm{CO}_{2}\right)$, metano $\left(\mathrm{CH}_{4}\right)$, helio $(\mathrm{He})$ y nitrógeno $(\mathrm{N})$ han sido documentados tanto de la laguna como del suelo adyacente al cráter del volcán; gases que aportan a la variabilidad climática pero sobre todo indican un probable ascenso de magma a través de fracturas o cambios de estrés en el sistema volcánicohidrotermal (Melián et al., 2010). 
Mapa 4. Indicador de afectación por gases volcánicos

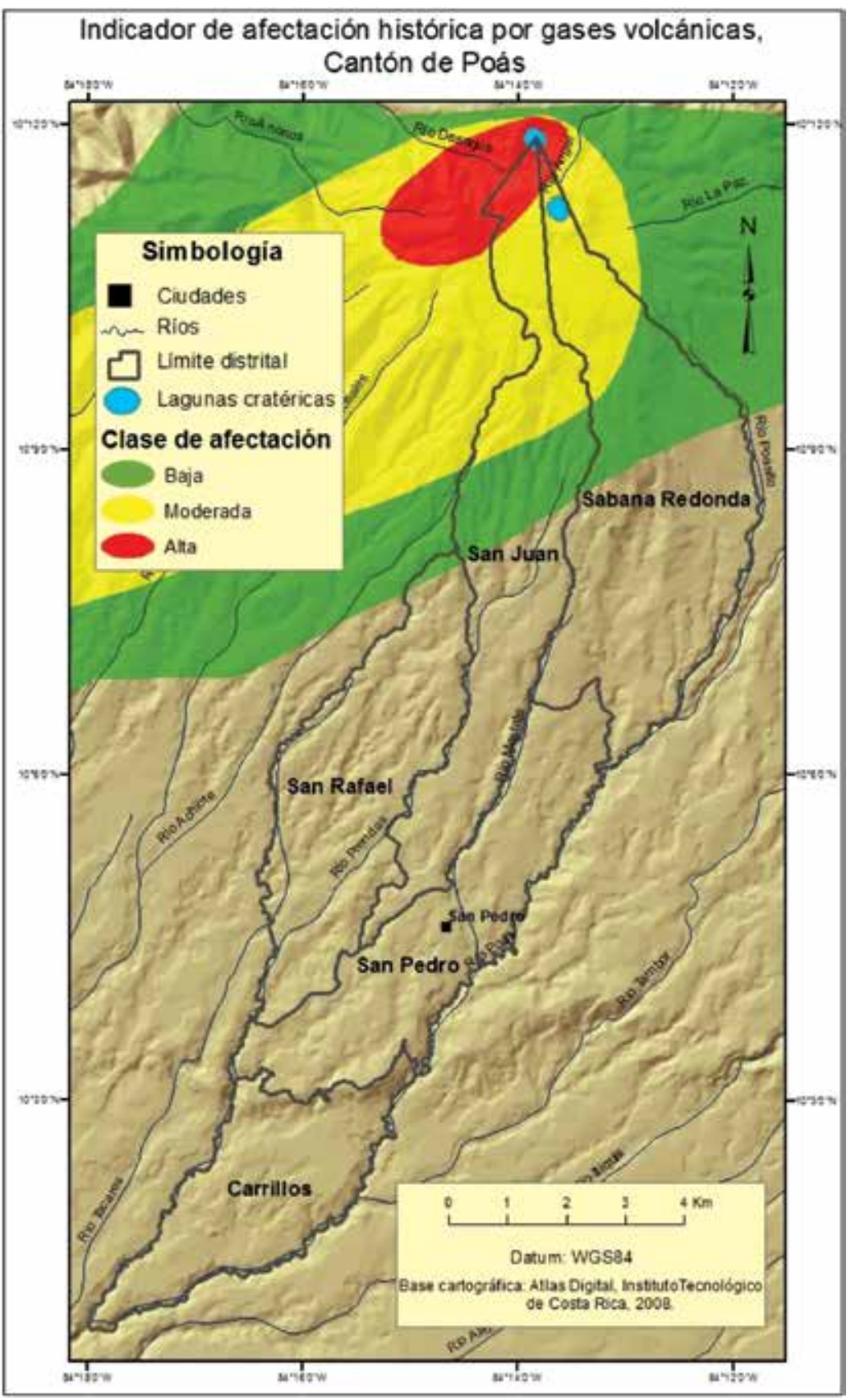

Fuente: elaboración propia 


\section{Afectación por terremotos}

Costa Rica se localiza sobre la placa Caribe y la microplaca de Panamá, en una zona tectónicamente activa producto de la cercanía con la zona de subducción, que produce sismicidad a lo largo de la Fosa Mesoamericana. Sin embargo, el registro instrumental no reporta eventos de origen interplaca que hayan afectado de manera considerable el área de estudio (posiblemente debido a la distancia de la zona de subducción) y por el contrario, se han presentado varios terremotos de tipo intraplaca. De acuerdo con Montero (2001) el área de estudio se ubica dentro del denominado Cinturón Deformado del Centro de Costa Rica, al oeste de la Cordillera Volcánica Central, donde predominan fallas normales y de rumbo, orientadas en patrones principalmente NW-SE, y N-S.

Los eventos sísmicos que más afectaron dicho cantón se resumen en el Cuadro 2. Dado el incipiente poblamiento de las laderas SW del Volcán Poás, en décadas anteriores, no se reportan importantes daños; sin embargo, el crecimiento demográfico reciente dio paso a mayores impactos debido al aumento de su vulnerabilidad.

Cuadro 2. Terremotos históricos en las cercanías del cantón de Poás

\begin{tabular}{|c|c|c|}
\hline Fecha & Título del evento & Observaciones \\
\hline 1772, Febrero, 15 & Terremoto de Barba & - \\
\hline 1888, Diciembre, 30 & $\begin{array}{l}\text { Terremoto de Fraijanes, } \\
\text { Alajuela }\end{array}$ & $\begin{array}{l}\text { Destrucción de Fraijanes, } \\
\text { deslizamientos en las laderas del volcán } \\
\text { Poás, } 6 \text { muertos. Desbordamiento de la } \\
\text { laguna de Fraijanes }\end{array}$ \\
\hline 1911, Agosto, 28 & $\begin{array}{l}\text { Terremoto de Toro } \\
\text { Amarillo, Grecia, Alajuela }\end{array}$ & Daños moderados y deslizamientos \\
\hline 1912, Junio, 6 & $\begin{array}{l}\text { Terremoto de Sarchí, Toro } \\
\text { Amarillo, Grecia, Alajuela }\end{array}$ & $\begin{array}{l}\text { Deslizamientos, avalanchas y } 7 \\
\text { muertos }\end{array}$ \\
\hline 1955, Septiembre, 1 & $\begin{array}{l}\text { Terremoto en Toro } \\
\text { Amarillo, Grecia, Alajuela. }\end{array}$ & 10 muertos \\
\hline 1992, Marzo, 6 & Sismo en Naranjo, Alajuela & $\begin{array}{l}\text { Magnitud } 6.0 \text { en Escala de Richter, } 1,5 \\
\text { km SE de Naranjo }\end{array}$ \\
\hline 2009, Enero, 8 & $\begin{array}{l}\text { Terremoto de Cinchona, } \\
\text { Alajuela. }\end{array}$ & $\begin{array}{l}\text { Magnitud } 6.2 \text { en Escala de Richter, } \\
\text { pueblo de Cinchona destruido, } 22 \\
\text { personas fallecidas, daños en ruta } \\
\text { nacional } 126 .\end{array}$ \\
\hline
\end{tabular}

Fuente: Basado en Vallejos et al. (2012) y Montero et al. (2010). 
Las incidencias de sismos históricos, se asocian de manera directa con los mapas de isosistas que han afectado el cantón, donde se registran valores VI, VII e inclusive VIII en el escala de Intensidades Mercalli Modificada (IMM), espacialmente en las cercanías del edificio volcánico.

De acuerdo con el indicador elaborado, las afectaciones considerables por movimientos sísmicos se presentan al norte del cantón (Mapa 5), asociados con pérdidas en la producción agropecuaria, así como de viviendas. Es importante también recalcar que los sismos en esta zona han disparado deslizamientos de varios tipos (traslacionales, rotacionales, caídas, desprendimientos y flujos de derrubios) que han contribuido a aumentar los daños; por ejemplo, en las líneas vitales (vías de comunicación, líneas eléctricas y acueductos) como ocurrió con el terremoto de Cinchona (Barrantes, Jiménez, \& Ocón, 2013). 
Gustavo Barrantes Castillo, Adolfo Quesada Román. Affectation cartography for natural hazards in Poás canton, Costa Rica

Mapa 5. Indicador de afectación por sacudida sísmica

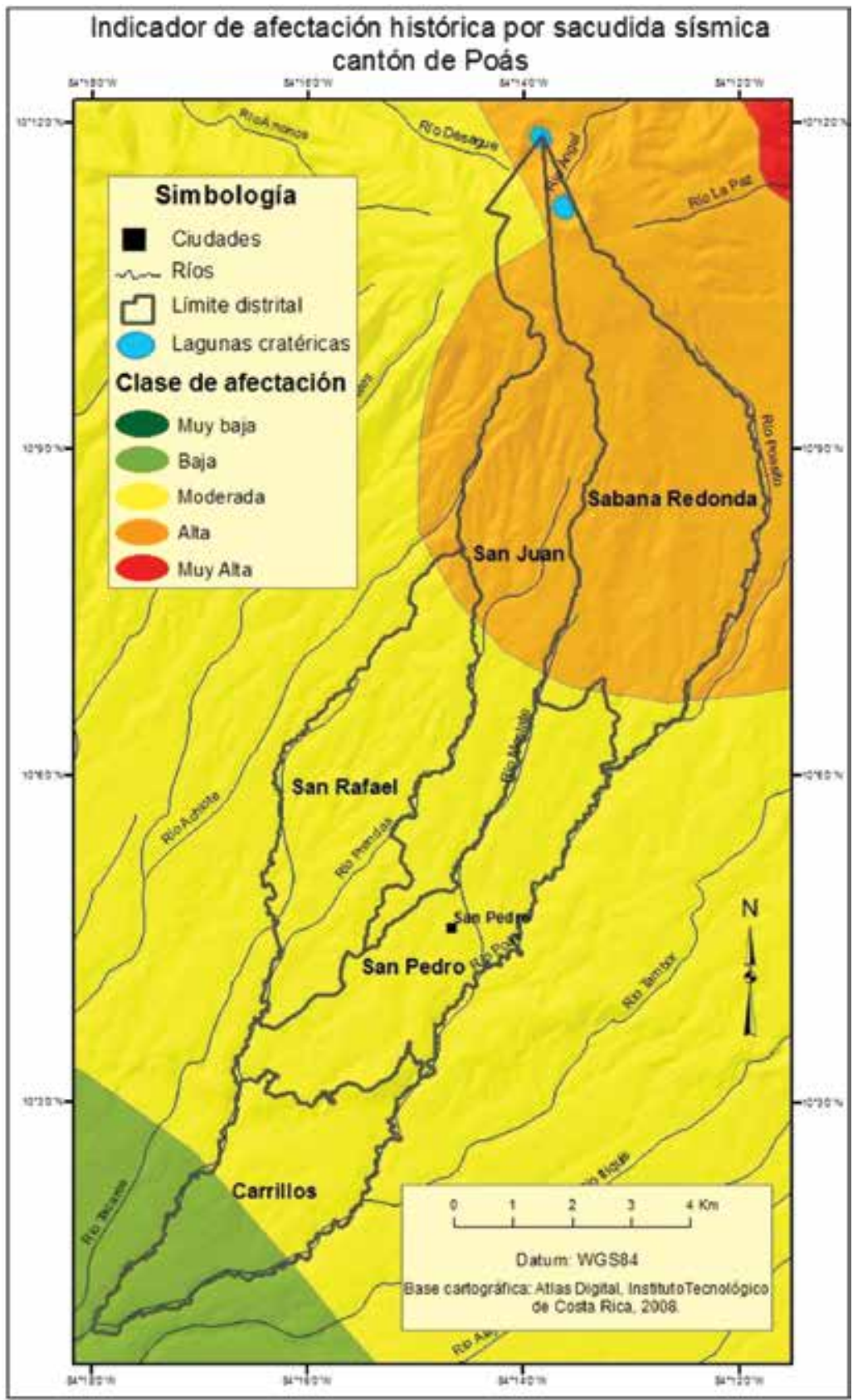

Fuente: elaboración propia 


\section{Afectación por inundaciones rápidas, basura y lahares}

De acuerdo con el mapa 6, los sitios donde se ha reportado mayores incidentes de inundación son tres: Sabana Redonda, San Pedro y Carrillos, que en conjunto representan el $79 \%$ de los casos registrados. Los demás eventos se presentan dispersos en el resto del cantón en poblados, tales como: Bajo Zamora, San Juan, San Juan Norte y San Rafael, con una recurrencia ocasional.

La causa principal de las inundaciones súbitas en el cantón de Poás son las lluvias intensas extremas en condiciones de suelos saturados durante la época lluviosa (de mayo a noviembre). Por su parte, la morfología fluvial del área de estudio se caracteriza por la presencia de valles profundos y en pocos casos con zonas de exiguas planicies de inundación, donde predominan los cañones debido a la disección de los cursos fluviales.

Según el registro, los cauces fluviales que presentan mayor incidencia de inundaciones son los ríos Poasito y Poás (sector de Sabana Redonda); río Mastate y Quebrada El Rastro (sector de San Pedro); y río Poás (sector de Carrillos). En el caso de los lahares la información disponible procede de la base de datos espacial de la CNE (CNE, 2015), información que no cuenta con metadatos o tablas que caractericen la información gráfica; por cuanto, solo permite identificar áreas que se supone corresponden con lahares ocurridos, estos se aprecian como canales rojos en el mapa 6. 
Gustavo Barrantes Castillo, Adolfo Quesada Román. Affectation cartography for natural hazards in Poás canton, Costa Rica

Mapa 6. Indicador de afectación por inundaciones rápidas y lahares

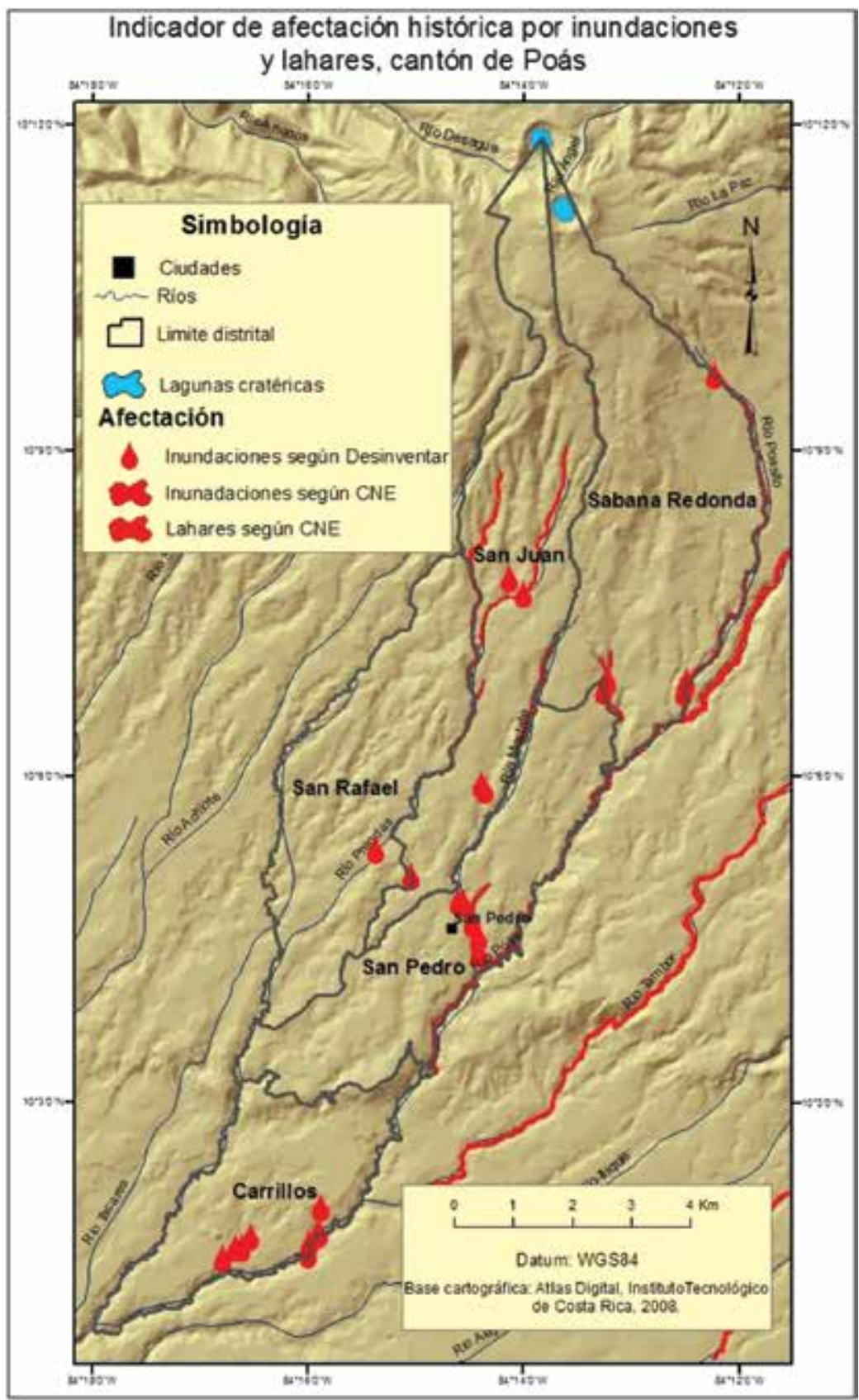

Fuente: elaboración propia 


\section{Afectación por deslizamientos}

En el cantón se identificaron 58 movimientos en masa, concentrados principalmente en dos áreas y el resto dispersos (Mapa 7), la primera se localiza al NE de la comunidad de San Juan Norte, en una franja de 3,5 km de largo (con orientación E-W) por $2 \mathrm{~km}$ de ancho (orientación N-S) que alberga 18 deslizamientos con áreas que varían desde los 622 hasta los 97 $470 \mathrm{~m}^{2}$. La segunda, se localiza a la altura del poblado de Sonora, donde se ubica un segmento del pliegue-falla de Alajuela con una orientación SW-NE, donde se distinguen 4 deslizamientos con áreas que varían entre los 7636 a $62110 \mathrm{~m}^{2}$. El resto de deslizamientos se encuentran dispersos en sectores próximos a poblados como: Guatuza, Potrero Chiquito y Santa Rosa en el distrito San Rafael y en Mastate y Altura en el distrito San Juan.

La mayoría de los movimientos en masa tienen como característica general localizarse en sustratos volcánicos cuaternarios como: coladas de lava, aglomerados, lahares o cenizas volcánicas (Kussmaul, 2000). Por lo general, se sitúan sobre pendientes fuertes que superan incluso los $35^{\circ}$ entre los interfluvios y el talweg así como con usos de la tierra predominantemente de agricultura o ganadería extensiva. La profundidad de la disección ronda los $40 \mathrm{~m}$, en la parte central del cantón y en la parte sur se presentan cañones de hasta $100 \mathrm{~m}$ de profundidad. 
Gustavo Barrantes Castillo, Adolfo Quesada Román. Affectation cartography for natural hazards in Poás canton, Costa Rica

Mapa 7. Indicador de afectación por deslizamientos

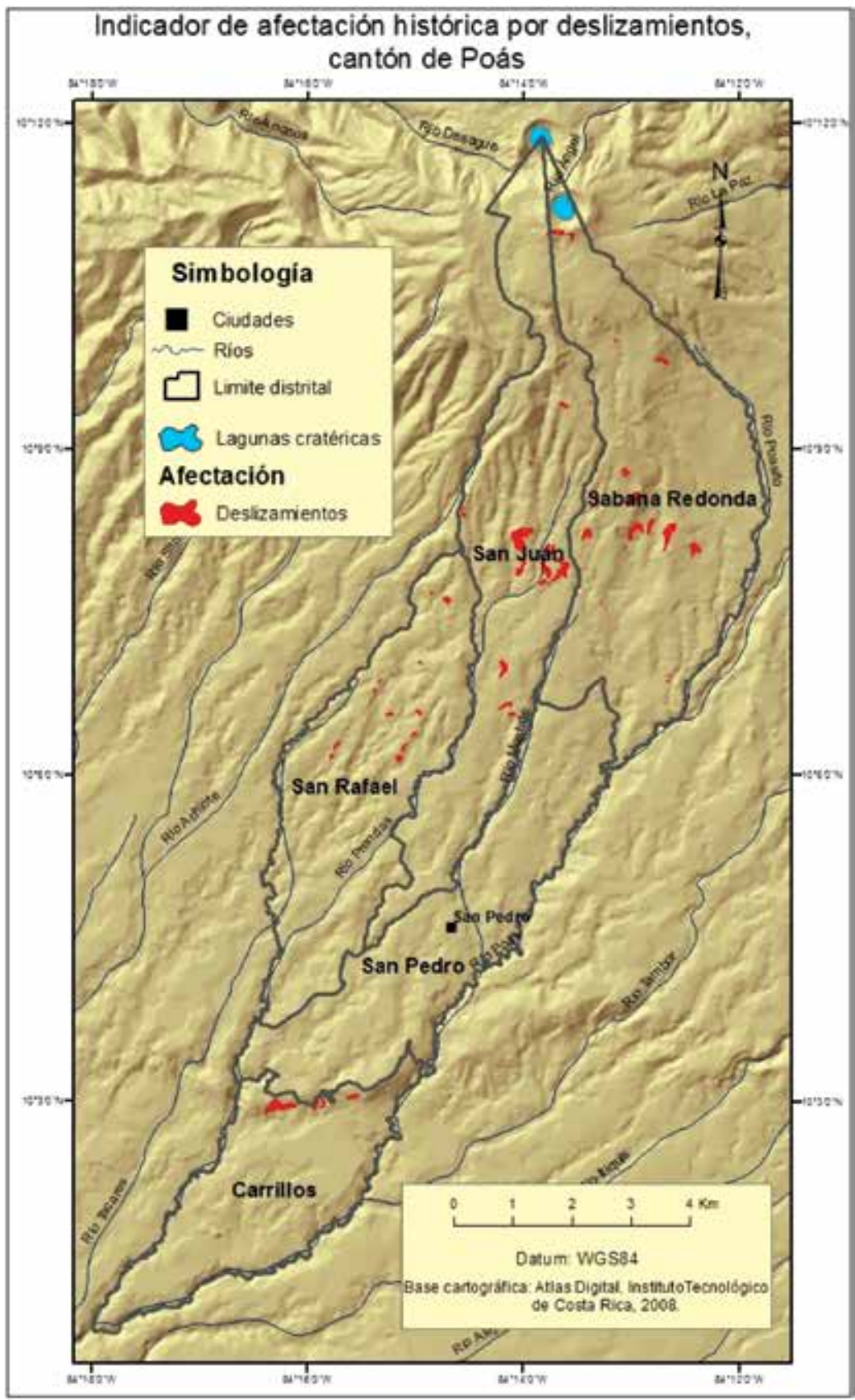

Fuente: elaboración propia 


\section{Afectación máxima por amenazas naturales}

En los mapas anteriores se presentó el resultado de la estandarización de las afectaciones ocurridas por tipo de evento, en las ventanas temporales a que se tuvo acceso por medio de los registros históricos, reportes técnicos, bases de datos y reconocimiento sobre modelos de elevación de alta resolución. El siguiente paso consiste en superponer las clasificaciones estandarizadas para todas las amenazas consideradas.

En el Mapa 8, se presenta la afectación máxima por eventos naturales (caída de ceniza, gases volcánicos, terremotos, inundaciones, lahares y deslizamientos), en él es posible apreciar que la afectación mayor (muy alta y alta en el mapa 8) se da en la parte norte del cantón, como resultado de su proximidad al cráter principal del Poás, la fuerte pendiente, elevadas precipitaciones de esa zona y la cercanía a fuentes sísmicas como: la falla Ángel y Sabanilla.

En términos generales, se observa que el nivel de afectación va disminuyendo de norte a sur (Mapa 8). En la parte central y sur del cantón se observa una afectación moderada como consecuencia de la atenuación sísmica por la distancia de las fuentes, la disminución del grosor de las capas de ceniza con la distancia al foco eruptivo y el hecho de no ubicarse en la dirección predominante de los vientos alisios para el caso de gases volcánicos. No obstante, tanto en la zona de alta como en la de moderada amenaza, se evidencia la presencia de parches de color rojo que corresponden con la clase de muy alta afectación (Mapa 8), estas áreas son el resultado de la distribución de los deslizamientos, inundaciones y lahares que se correlacionan espacialmente con valles encañonados y laderas de fuerte pendiente.

Finalmente, en el extremo sur del cantón la afectación máxima histórica resulta baja a consecuencias de la mayor distancia de las fuentes volcánicas y sísmicas, así como la menor pendiente y humedad del terreno; no obstante, se reportan inundaciones en el área urbana de Carrillos. En términos generales, puede afirmarse que se presenta una amenaza natural entre muy alta a alta en los distritos de San Juan y Sabana Redonda, moderada en San Rafael, San Pedro, la sección sur de San Juan y la sección norte de Carrillo y baja en la sección sur de Carrillo. 
Gustavo Barrantes Castillo, Adolfo Quesada Román. Affectation cartography for natural hazards in Poás canton, Costa Rica

Mapa 8. Indicador afectación por las amenazas naturales del cantón

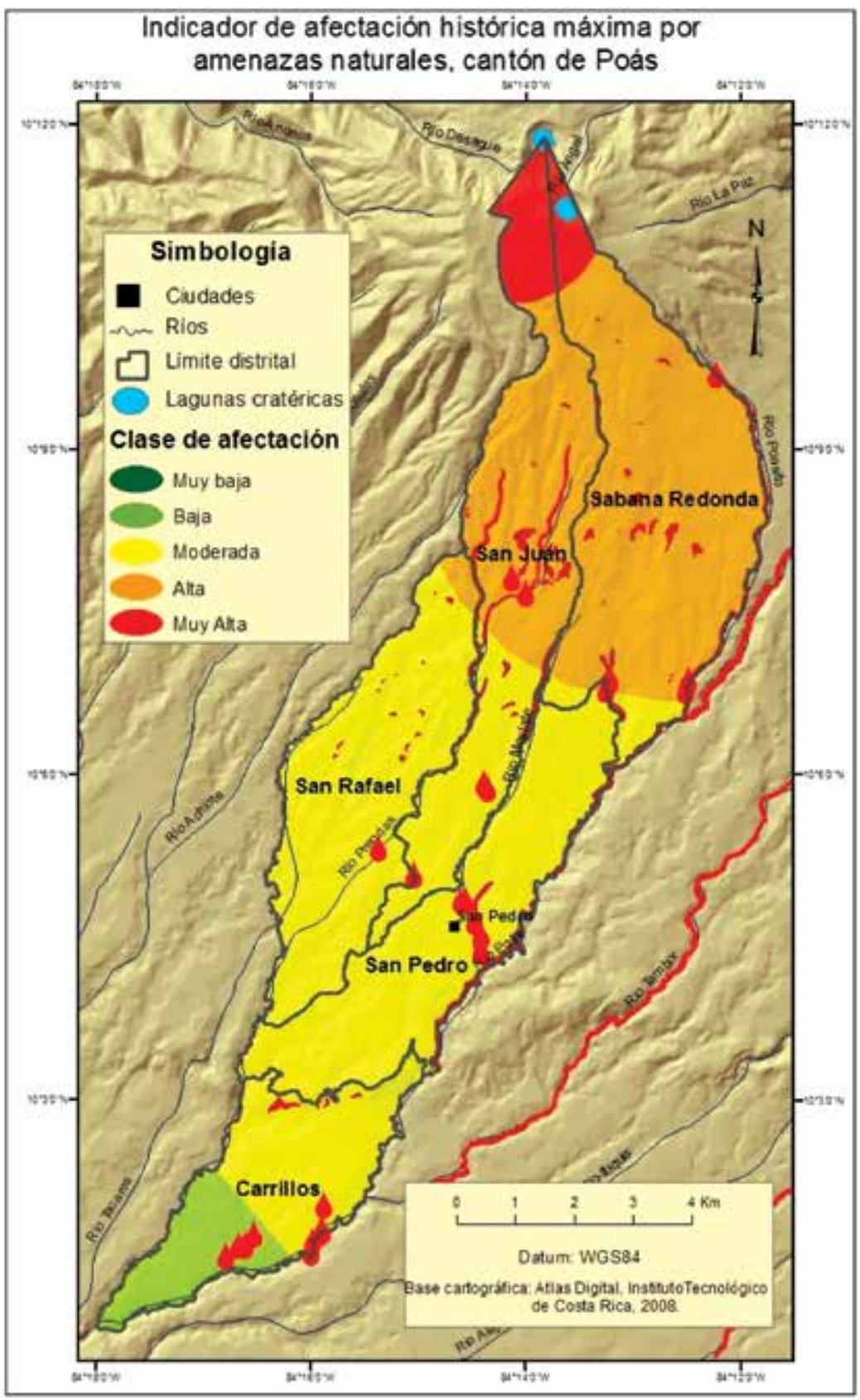

Fuente: elaboración propia 
Cabe resaltar que el Mapa 8 representa la máxima afectación por eventos registrados y en ningún caso debe obviarse que la intensidad de los eventos naturales o el aumento de la vulnerabilidad pueden variar sensiblemente la distribución planteada. Sin embargo, la información generada es pertinente para retroalimentar los procesos de Ordenamiento Territorial (OT) y gestión del riesgo, además de complementarse con escenarios futuros.

\section{Discusión de resultados}

A pesar de la existencia de bases de datos, reportes, recopilaciones y documentos históricos, el registro de daños por tipo de evento resulta incompleto, generalmente, impreciso y en muy pocas ocasiones georreferenciado o cartografiado. Las excepciones a esta condición son los eventos recientes que cuentan con reportes técnicos (en particular los sismos para los que generalmente están disponibles mapas de isosistas), pero no en todos los casos, ya que son frecuentes los reportes de la CNE que no precisan una dirección con coordenadas geográficas.

La modelación de amenazas naturales por métodos tanto cualitativos como cuantitativos, requiere de la existencia de datos que faciliten su calibración y en algunos casos permitan su validación. Si bien esta tarea no se realizó correctamente en el pasado, a pesar del esfuerzo realizado por González-Víquez (1910), Arroyo \& Patterson (1988) y Vallejos et al. (2012), la falta de una visión espacial en la recolección y almacenamiento de los datos actuales, como lo realiza Desinventar, solo ha permitido disponer datos con valor estadístico para analizar unidades administrativas como los cantones o distritos. La base de datos espacial de la CNE tampoco solventa el problema al no incluir información alfanumérica y al no disponer de metadatos.

El trabajo realizado en esta investigación es pionero en Costa Rica y pretende hacer reflexionar a las autoridades e investigadores sobre la importancia y conveniencia de tener datos estandarizados y georreferenciados de daños; así como afectaciones por eventos naturales, con el fin de utilizar dicha información en procesos de ordenamiento territorial y gestión del riesgo.

El conocimiento de los sitios que han sido afectados por amenazas naturales debe considerarse como un elemento restrictivo al uso urbano en el OT. En otras palabras, es posible utilizar la información generada para 
hacer prospección del riego, de manera que se asignen usos compatibles con el nivel máximo de afectación conocido en los Planes Reguladores, como podría ser agricultura extensiva o protección natural.

\section{Conclusiones}

Las amenazas naturales más frecuentes en el cantón de Poás son las inundaciones y los deslizamientos; sin embargo, su área de afectación resulta más puntual o reducida. Por su parte, el vulcanismo y la sismicidad son las amenazas que abarcan mayores áreas de afectación; por ejemplo, la afectación por gases volcánicos ocurrida en 1968, 1989 y 1994 o los terremotos de Fraijanes de 1888 y Cinchona de 2009.

La sección norte del cantón, sobre los distritos de Sabana Redonda y San Juan, resulta ser el área de mayor afectación histórica debido a su proximidad con el cráter principal del Poás y a las fallas activas de Ángel y Sabanilla.

En la parte intermedia del cantón (distritos de San Rafael, San Pedro), se desarrolla un amplio piedemonte irrigado por ríos como: el Poás, Prendas y Mastate, que dadas sus condiciones de pendiente y litología favorecen el desarrollo de deslizamientos en las laderas de los valles e inundaciones rápidas que son contendidas por la profunda disección de los cursos fluviales.

En la parte sur del cantón (Carrillos), la mayor afectación se relaciona con inundaciones dado el cambio de pendiente y la confluencia de ríos tributarios del Poás, así como por deslizamientos relacionados al plieguefalla de Alajuela.

\section{Referencias}

Arroyo, L. N. \& Lavell, A. (1991). Costa Rica: Zonas de riesgo, vulnerabilidad física y social. In Costa Rica: Zonas de riesgo, vulnerabilidad fisica y social. Costa Rica. Universidad Nacional (UNA). Escuela de Ciencias Geográficas.

Arroyo, L. N. \& Patterson, O. (1988). Tipos y distribución de algunos peligros naturales en Costa Rica (Sismicidad y vulcanismo, deslizamientos, sequías e inundaciones). Trabajo de Investigación $\mathrm{N}^{\circ}$ 822066. Heredia, Costa Rica. 135p. 
Barquero, J. \& Duarte, E. (1990). Erupciones de gases y sus consecuencias en el volcán Poás, Costa Rica. Boletín de Vulcanología (21), 13-17.

Barrantes, G., Jiménez, C., \& Ocón, M. J. (2013). Deslizamientos provocados por el Terremoto de Cinchona, Costa Rica. Revista Geográfica de América Central, 51, 89-100.

CENAPRED. (2001). Estabilidad de laderas. Serie Fascículos, 1-36.

Casertano, L., Borgia. A. \& Cigolini, C. (1983). El Volcán Poás, Costa Rica: cronología y características de la actividad. Geofísica Internacional, 22 (3), 215-236.

CNE. (2008). Atlas de amenazas naturales de Costa Rica. San José, Costa Rica. Disponible en: http://www.cne.go.cr/

CNE. (2015). Base de Datos Espacial sobre amenazas naturales de Costa Rica. San José.

Denyer, P. \& Kussmaul, S. (1994). Atlas geológico de la Gran Area Metropolitana, Costa Rica: Editorial Tecnológica de Costa Rica.

Duarte, E., \& Fernández, E. (2011). Callejones de acidificación en los tres volcanes activos de Costa Rica. Revista Geográfica de América Central, 47, 127-130.

EM-DATA. (2015). Recuperado de International Disaster Database: http:// www.emdat.be/database

Gazel, E. \& Ruiz, P. (2005). Los conos piroclásticos de Sabana Redonda: Componente magmático enriquecido del Volcán Poás, Costa Rica. Revista Geológica de América Central, 33, 45-60.

González-Víquez, C. (1910). Temblores, terremotos, inundaciones y erupciones volcánicas en Costa Rica. San José: Tipografía Avelino Alsina.

INEC. (2012). Censo 2011 Resultados. Recuperado de: http://www.inec. go.cr/Web/Home/GeneradorPagina.aspx

Kussmaul, S. (2000). Estratigrafía de las rocas ígneas. En: Denyer, P., Kussmaul, S. (Eds). (2000). Geología de Costa Rica. Editorial Tecnológica de Costa Rica. Cartago, Costa Rica. 63-86

LA RED. (2015). DesInventar. Recuperado de http://online.desinventar.org/ Melián, G.V., Pérez, N. M, Hernández, P.A., Nolasco, D., Marrero, R., Fernández, M., Ramírez, C., Mora-Amador \& Alvarado, G.E. (2010). Emisión difusa de $\mathrm{CO} 2$ y actividad volcánica en el volcán Poás, Costa Rica. Revista Geológica de América Central 43, 147-170. 
Montero, W. (2001). Neotectónica de la región central de Costa Rica: frontera oeste de la microplaca de Panamá. Revista geológica de América Central, 24, 29-56.

Montero, W., Soto, G., Alvarado, G. \& Rojas, W. (2010). División de deslizamiento tectónico y transtensión en el macizo del Volcán Poás, basado en estudios neotectónicos y de sismicidad histórica. Revista geológica de América Central, 43, 13-36.

Montero, A. \& Salazar, S. (1991). Los desastres en Costa Rica. Comisión Nacional de Emergencias. San José, Costa Rica. 25 pp.

Mora, R. (2010). Peligrosidad volcánica del Poás, basado en las principales erupciones históricas de 1834, 1910 y 1953-1955. Universidad de Costa Rica: Tesis para optar al grado de Máster en Gestión de Riesgos de Desastres y Atención de Emergencias.

Morales, L. D., Montero, W., \& Madrigal, R. (1992). El Terremoto de Fraijanes de 1888 y el sistema de fallas de Alajuela: implicaciones del peligro sísmico potencial para el Valle Central. Revista Geográfica de América Central, 25-26, 227-240.

Ordaz, M. (2007). Información para la gestión de riesgo de desastres. Estudio de caso de cinco países. Ciudad de México: CEPAL-BID.

Prosser, J. T. \& Carr, M. J. (1987). Poás volcano, Costa Rica: geology of the summit region and spatial and temporal variations among the most recent lavas. Journal of volcanology and geothermal research, 33, 131-146.

Rojas, W., Montero, W., Soto, G., Barquero, R., Boschini, I., Alvarado, G. \& Vargas, A. (2009). Contexto histórico, geológico, tectónico loca, sismicidad histórica y registro sísmico instrumental. En R. Barquero, El terremoto de Cinchona, 8 de enero de 2009 (págs. 7-33). San José: RSN UCR-ICE.

RSN (2009). El terremoto de Cinchona del jueves 8 de enero de 2009. Revista Geológica de América Central, 40, 91-95.

RSN: UCR-ICE. (2014). Volcanes de Costa Rica: Datos del volcán Poás. Recuperado de http://www.rsn.ucr.ac.cr/index.php/vulcanologia/ informacion-general/31-poas

Vahrson, W-G., Arauz, B.I., Chacón, B.R., Hernández, G. \& Mora, S. (1990). Amenazas de inundaciones en Costa Rica, América Central; comentarios al mapa 1:50000. Informe a la Comisión Nacional de 
Emergencias (CNE) y al Centro de Prevención de Desastres Naturales en América Central (CEPREDENAC). Heredia, Costa Rica. 66 pp. Vallejos, S., Esquivel, L. \& Hidalgo, M. (2012). Histórico de desastres en Costa Rica (Febrero 1723 - Setiembre 2012). San José, C.R.: CNE, 2012. Vargas, C. (1979). Antología el volcán Poás. San José: EUNED 